\title{
COGNITIVE INFORMATION SYSTEMS - ARTIFICIAL INTELLIGENCE \& MANAGEMENT DECISIONS
}

\author{
Bálint Molnár and Dóra Mattyasovszky-Philipp \\ Information Systems Department, Eötvös Loránd University, ELTE, \\ Pázmány Péter Sétány 1/C, Budapest, Hungary
}

\begin{abstract}
We investigate the interlink between the management decisions and Artificial and Computational Intelligence (ACI) in the context of Cognitive Information Systems (CIS). The analysis is focused on the current trends of application of ACI within the realm of Information Systems (IS) for supporting management decision. One of the research issues is whether there is a difference between CIS and IS based on Artificial Intelligence (AI). Is there any chance to distinguish them from each other? If yes, we should locate the subtle differences and similarities between them. There are various definitions for the recent and the future AI, there are approaches to circumscribe the concept of CIS. The research issue is how the Cognitive Architecture, Science, IS and AI can be reconciled for management information systems (MIS) to support decision making.
\end{abstract}

\section{KEYWORDS}

Cognitive Information Systems, Enterprise Information Systems, Cognitive Architecture, Artificial Intelligence, Computational Intelligence, Management Decision

\section{INTRODUCTION}

MIS, Enterprise Resource Planning Systems (ERP) and their spin-offs - as Customer Relationship Management Systems (CRM) etc. - play important role within enterprises for supporting the management in decision making whereby these systems occupy an important position within Management Sciences as well. The most recent trends in ACI, Machine Learning (ML), Data Science, which are placed under one hood of AI, provide enormous selection of tools for exploiting data, content that are stored in IS. However, there are preconditions for both using and understanding of data (Rasmussen, 1980), (Mattyasovszky-Philipp \& Molnár, 2018) ."Three important concepts help make a system cognitive: contextual insight from the model, hypothesis generation (a proposed explanation of a phenomenon), and continuous learning from data across time." (Hurwitz, 2015.) Extending Hurwitz (2015) definition of CIS, the ideal CISs, other than the above predefined details, should impact the carbon agent cognition in a positive way, improving it, leveraging the synergy originated form he interactions between silicon and carbon agent. This synergy generated leverage expressed via cognitive resonance, where the cognitive resonance orienting the entire process to the automated data understanding, meanwhile extracting the semantic information, which supporting the interpretation of the understanding. The concept of cognitive resonance is one of the attempts that try to make sense of the modelling activities in the most recent world of data analytics that uses tools out of data science (Mattyasovszky-Philipp \& Molnár, 2018). CIS related information and the knowledge is limited, the lack of information generates misunderstandings, that has to be clarified for further explanation. Therefore the aim and the expected contribution of the research is to find a model, and combine the available knowledge considering CIS and management decision, and then to analyse the gap within silicon and carbon agent interaction from management decision prospective, to support the understanding, therefore support the enterprise operation using CIS. The research methodology that were applied during the research analyses and assessment of the available (limited) publications pursued by the comparative study pattern, i.e. studying and comparing the results published in articles, then a case study paradigm has been used for observing and monitoring the work with a CIS during daily operation (Molnár \& Mattyasovszky-Philipp, 2018). 


\section{MODEL FOR DECISION MAKING IN CIS}

A model is a representation of a system of "relevant" properties assumes an oracle. The system may consist of one or more entities and their relationships that are the relevant entities that should own at least two distinctly definable properties that can and should be related to each other (Muller et al., 2012).

Definition: Models in computer science and informatics aim to capture semantic properties of a domain of discourse by syntactic representations for the pragmatic benefits of users. A model is a tuple $M=(W, R, I: W \rightarrow R)$, (1) where $W$ is the modeled system (domain of discourse); (2) $R$ is the representation of modeled system $W$; (3) $I$ is an interpreter that determines the semantic properties of $W$ in terms of syntactic expressions of $R$. To build up a model $M$ means to specify $W, R, I$ and to define the mapping $W \rightarrow R$. To describe $R$ means to determine the purpose of the model that it should serve (i.e. to acquire knowledge of the structure, of patterns, of behavior, of functionality of the system); furthermore, the representation $R$ defines the entities that constitute the model and the set of relationships thereby the significant parameters of the properties can be identified. The interpreter $I$ assists the human being, the carbon agent to understand the meaning of the syntactical expressions, data, bit strings, signs. Basically, the interpreter I can evaluate logical formulae whether they are true or false then more complex and meaningful description can be constructed from these elementary statements for understanding (see Herbrand Universe. (Coppin, 2004)).

\subsection{Interaction between Managers and Cognitive Systems}

In a Business Cognitive Information Systems, we meet two types of models. One model is that describes the human computer interaction, i.e. information exchange between silicon agent and carbon agent. The other type of models is that is built up for training through the available data and the model is associated to a certain algorithm belonging to the realm of ACI and ML. Such models out of data science approaches are the subject of interaction between humans and machines. In the environment of CIS, one of the helping hand for modeling the interaction between the carbon agent and silicon agent is the cognitive resonance (Ogiela, 2010). The services for data science and data analytics that are offered are based on the following pattern: (1) Creation of data collections of historical data. (2) The data set will become the basis for training the selected algorithm. (3) The problem should be defined, and experiments should be started to seek the proper models and algorithms measured in appropriate metrics whether it fits to purpose. (4) Afterward the models should be finalized so that it should yield good approximation on the training data set and according to the pre-defined accuracy metrics. (5) Finally, the models could be used for prediction and prescription for the enterprise (Wirth \& Hipp, 2000), (Clarke et al., 2009). The MIS environment can be characterized by the notion of "Big Data" and "AI." Disregarding hype, there are serious problems that should be handled within the modeling of manager-CIS interactions. Modeling carbon agent - silicon agent information interchange means that two sets of parameters on both sides should be clearly defined, the two sets of parameters embodying single models should be related to each other. The parameters implicitly formalize models on both sides, in data science and analytics context means that the data that are required for decision making should be organized into analytics base table $(A B T)$. The well-formed ABT integrate the disparate data and information sources that are available within the IS of the enterprise and the ABT will be the subject of the various algorithms that may produce predictions and IS prescriptions for the organization. The ABT and a set of algorithms together form the model. However, the parameters on the silicon agent side are defined with respect to the symbolic representation of the interface, and this model described by parameters does not reflect the representation of underlying structure within the machine. Since, there are lots of alternative user interfaces that represent the underlying data science models whose parameters at interface levels should be mapped and correlated to parameters that are apt to the human abilities. In a CIS environment, carbon agents are components of the system during the man-machine communication. The carbon agent should build up a representation of data science models' structure and acquire understanding the function and algorithms of the models with respect to a set of tasks connected to decision making. The carbon agent should learn the description language of the data science model, i.e. the symbols, signs, denotations that are employed, the syntactical rules, the essence of operations and algorithms that belong to these elements, those operators that can be used to invoke services for manipulating the models. The sequences of operations are related to tasks and subtasks of business processes in the enterprise. The cognitive model within the mind of carbon agent is a mapping of a virtual machine that is a contracting map of components that embrace the user interface with operations, the data science models along with data structure and algorithms, and the computing machinery. 


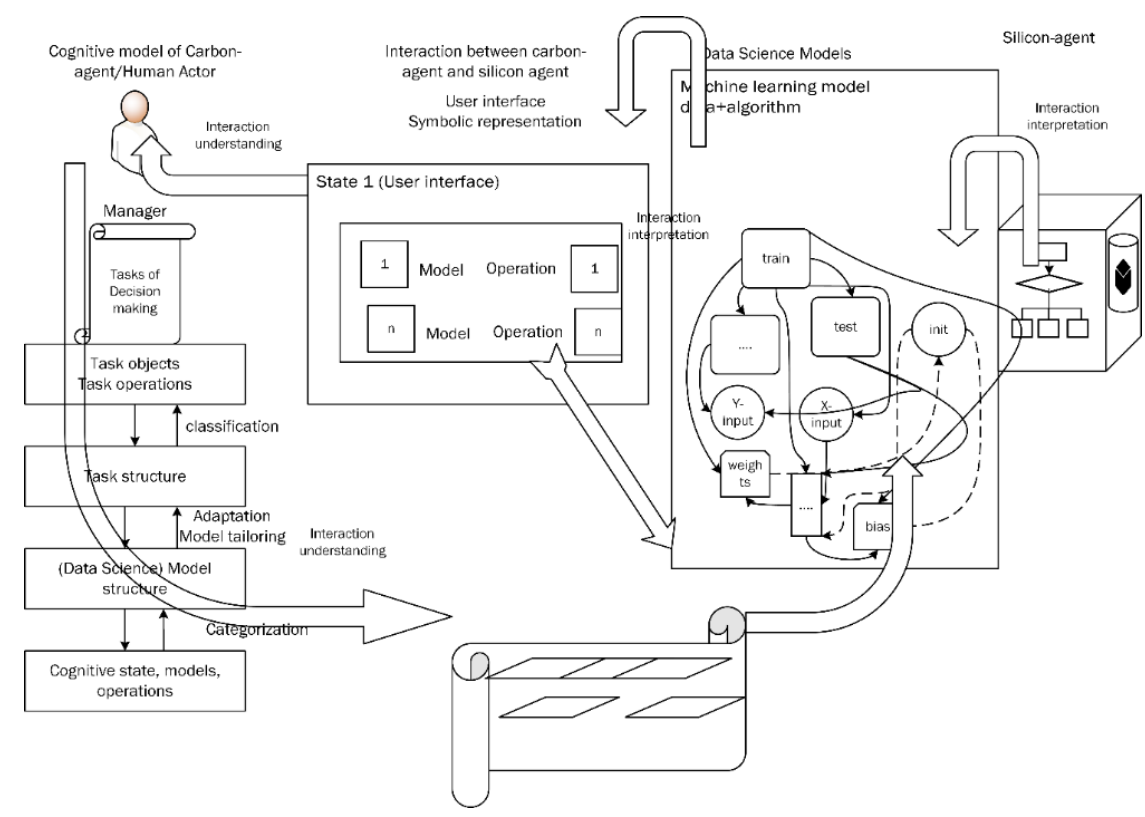

Figure 1. Model for the Interaction between Carbon Agent and Silicon Agent

The most important phenomena for the end-users are the relationships between tasks within business processes and the services of silicon agent including user interface, data science model and the underlying complex IS. On modeling the carbon agent and silicon agent information interchange, the system should take into account the symbolic representation of data science models, cognitive models of carbon agent, the mental models in the mind of the end-users, and the complex relationships among them (Figure 1).The symbolic representation of the virtual machine consists of the following elements: (1) objects that are manipulated by operations, (2) operations that are depicted by symbols and their syntactic rules and cause changes states of the systems, (3) states of the system that can be characterized by the permitted operations and set of significant parameters. The cognitive model that captures the mental representation of the environment within the mind of carbon agent contains the following constituents: (1) objects, (2) operations, (3) states and state transitions, (4) architecture (business, information, application system), (5) tasks and their structure of business processes.

\subsection{Strategic Management Decision \& CIS}

The process of the decision making a consist of multiple perceptions and many other, sometimes the result of the decision leads in the mistaken way. Boulding (1994) try to close the gap between the actual and normative managerial behavior researching the strategic decision process. Strategic managerial decision messier, they occur in a complex environment difficult or expensive to reverse and the outcomes are to a greater degree contingent, that are influenced by individuals and impacted by the organization's behavior, and often substantially alters the internal and external relationships. The viability of the decision depends on the knowledge about the current status and about the expected reactions. Management's decision in its surrounding environment, while this environment surrounds the organization (Boulding, 1994). The decision making starts with a trigger, which before the outcome could originate a start new decision process. the model highlights two questions, "what" and "how"? The framework measuring the manager knowledge and his believes about the current economic environment, which determinates the value assigned to the decision. "What" knowledge determines the value that manager assigns to the mental market model, which represents the facts. The "how" as a manager mapping function represents his/her current believes about the market behavior. "How" impacted by multiple factors, external and internal, from price sensitivity to the personal relationship etc., meanwhile the manager "What" and "how" decision and knowledge about the situation linked to the "if-than" decision rule. The "if-than" knowledge, must be at least implicitly generate by some managers "how" knowledge. "What", and "how" contains, the anticipated outcome as a believe of the manager, because the manager maps the context and the anticipated reaction within his/her mind. There are 
highlighted tree major reasons, when 'what" may differ to the actual situation: (1) Wrong mental (market) model: wrong variables and/or functional forms etc., (2) Omitted variables (unobserved, ignored facts), (3) Wrong weights included into the decision model. All of them might have a significant impact on the outcome of the decision. Context and the it's change impact the relative sensitivity of the trigger mechanism. These contextual factors influence the overall mental model of the manager, therefore the decision process impacted thru the outcome. Managers might learn from the decision process, which does not imply better or the right decision. Meanwhile, because of the confusing strategic decision-making process it is possible that the managerial knowledge deteriorates rather than increase over the time. According to the decision research the problem decomposition increase the quality of the decision (Boulding, 1994). Managers are humans, sometimes, the decision taking is correct, but might happen it's opposite. The technology is able to mimic the human decision-making process in general, however there is an uncertainty whether the individual decision-making remains always individual process, considering the above "mental" model. We examine what we could expect from those systems and how we could use them to support the management decision. Those are the questions to be answered to be able to better understand CIS in the light of the "mental" model, considering the process of strategic decision making. "What": (1) CIS is capable to (after appropriate coding and programming) use the correct "mental" (market) model. (2) CIS never omits variable or ignore available data or facts, and it apply the weights coded. (3) Advanced technology ensures the learning cycle, a supervised learning ensure the highest possible accuracy of the collected knowledge, which impact the system output. "How": (1) CISs are not yet trained to consider the part of anticipation of reactions, which is included into the human strategical decision process.

Silicon and carbon agent interaction with supervised learning is one possible solution, however the anticipation related to the reaction is still to be built in to CIS, to empower CIS to mimic the human strategical decision-making process. Potential development as the carbon agent is learning from the previous decision process, might be included for one scenario, but in a different situation - stating that the subject of the prediction is different - this learned adds in is not available, therefore this gap in the decision-making process seems to be standard. That causing a chain of chains as iterations, to a continuous process. It may be simple, but this process could be repeated as many times as a difference occurs from the original concept, i.e. the anticipated reaction. This would appear weighted in the result obtained; as changing one's expectations on one side, approaching the "ideal of final" anticipation would shape the ending output. On the other hand, carbon agent repeats any mistakes resulting from a previous decision-making. Most probably the above described exercise to train any CIS to be able to consider expectations is not affordable, from time prospective. Any change in the environment might re-trigger a need of new training, that takes further form the acceptable output. (Acceptable result is key during managerial decision, due to various limitations exits in the business environment.) However, the complexity might be managed easier, speed of the environment change might impact negatively the learning cycle -lack of time to re-train, therefore the decision/output might not be accurate. To this end, it is closely related to the fact that the delegation levels associated with each job role are not accidentally defined within an organization.

\subsection{Research \& CIS with Cognitive Resonance}

We consider that cognitive resonance is an additional feature of CIS, due to speed up the expert knowledge of CIS, support management decision with the newly learned information, and it improves during iteration by iteration providing new aspects in line with the decision taken. The current research in AI related in knowledge representation focuses on restricted logics that guaranty the efficient processing, meanwhile there are less flexibility and power that found in the human reasoning (Langley, 2015). Our view the focus of the restricted logic is extended, balanced and corrected in sense of quality improvement and improvement of the features of CIS during the cognitive resonance. The power ensured by the cognitive resonance as a" power" or knowledge transfer via learning cycle. During the problem solving and planning relies on extensive search and emphasize processing speed, and bears little resemblance to flexible problem solving in humans" (Langley, 2015). The carbon-silicon cooperation strictly focuses on the problem solving, meanwhile narrows and synthesizes the area of search, which supports the existence of cognitive resonance. Researches in case of Natural Language Processing the current trend of the AI research focus on statistical methods with few links to psycho/linguistics and emphasis on tasks like information retrieval and extraction (Langley, 2015). The already solved problems utilizing the synergy originated via cognitive resonance, where stored as a "personalized" knowledge in relation to the already learned problem, therefore all inputs and outputs 
including all search result are available for the future as a "template" rules of the problem solution, which significantly improve the speed of the data and information elaboration for further problem solving. The expert based supervised learning supports the template (pattern) generation, speed up the problem solving to exclude all not appropriate, or necessary data. Considering ML, the trend is focuses on statistical techniques that learn far more slowly than humans and almost exclusive focus on classification and reactive control (Langley 2015). The silicon carbon collaboration, speeds up the learning cycle, the understanding and the implementation of any statistical technique, as the classification and the reactive control as well. Our proposal is that these research emphases should be supplemented and evaluated together with the interpretation of cognitive resonance.

\section{CONCLUSION}

We have overviewed the most recent literature on CISs related to business administration and decision making. The definition of CISs refers to the cognitive architectures, data processing, models for insights hypotheses for new phenomenon and opportunity for the silicon agents for the continuous learning. The cognitive resonance provides a kind of interpretation framework for describing the information interchange between human and automated systems. Although, the ideas of economic analysis are superficial from point of operating enterprises where the management and IS should solve in tandem serious problems. Hence, the research has plenty to do on defining the idea of CISs in economics, in business administration as a management decision support system, and in the organization science to leverage the tools of data science for business efficiency and effectiveness during decision process.

\section{ACKNOWLEDGEMENT}

The project has been supported by the European Union, co-financed by the European Social Fund (EFOP-3.6.3-VEKOP-16-2017-00002).

\section{REFERENCES}

Boulding, W. e. a., 1994. Understanding managers' strategic decision-making process. Marketing Letters, 5(4), pp. 413-426.

Clarke, B., Fokoue, E. \& Zhang, H. H., 2009. Principles and theory for data mining and machine learning. Dordrecht Heidelberg London New York: Springer Science \& Business Media.

Coppin, B., 2004. Artificial intelligence illuminated. Sudbury, MA, US: Jones and Bartlett Publishers.

Hurwitz, J. K. M. B., 2015. A Cognitive Computing and Big Data Analytics., 10475 Crosspoint Boulevard Indianapolis, IN 46256.: John Wiley \& Sons, Inc.

Langley, P., 2015. Introduction to Interactive Cognitive Systems, Auckland: University of Auckland.

Mattyasovszky-Philipp, D., 2018. Research on Cognitive Information Systems in Enterprise Environment. Moscow, Russia, CEUR, pp. 232-237.

Mattyasovszky-Philipp, D. \& Molnár, B., 2018. Cognitive Enterprise and Cognitive Information Systems. Szeged, Hungary, Polygon Publisher, p. 64

Molnár, B. \& Mattyasovszky-Philipp, D., 2018. Cognitive Information Systems and Enterprise Engineering. Luxembourg, CEUR Vol-2229.

Muller, P.-A., Fondement, F., Baudry, B. \& Combemale, B., 2012. Modeling modeling modeling. Software \& Systems Modeling, 11(3), pp. 347-359.

Ogiela, L., 2010. Cognitive informatics in automatic pattern understanding and cognitive information systems. In: Advances in Cognitive Informatics and Cognitive Computing. Berlin, Heidelberg: Springer, pp. 209-226.

Rasmussen, J., 1980. The human as a systems component. In: Human interaction with computers. London: London Academic Press, pp. 67-96.

Wirth, R. \& Hipp, J., 2000. CRISP-DM: Towards a standard process model for data mining. Manchester, UK, Practical Application 2000, pp. 29-39. 